\title{
Towards an asset-basedapproach to promoting and sustaining well-being for people with aphasia and their families: an international exploratory study
}

Ciara Shiggins $^{1}$, Varda Soskolne ${ }^{2}$, Dafna Olenik ${ }^{3}$, Gill Pearl ${ }^{4}$, Line Haaland-Johansen ${ }^{5}$, Jytte Isaksen $^{6}$, Caroline Jagoe ${ }^{7}$, Ruth Mc Menamin ${ }^{8} \&$ Simon Horton $^{1}$

${ }^{1}$ School of Health Sciences, University of East Anglia, Norwich, UK

${ }^{2}$ School of Social Work, Bar-Ilan University, Ramat Gan, Israel

${ }_{3}^{3}$ Adler Aphasia Centre, Hadassah Academic College Jerusalem, Israel

${ }^{4}$ Speakeasy, Bury, UK

${ }^{5}$ Department of Speech and Language Disorders, Statped, Oslo, Norway \& Faculty of Education and Arts, Nord University, Bodø, Norway

${ }^{6}$ Department of Language and Communication, University of Southern Denmark, Odense, Denmark

${ }^{7}$ School of Linguistic, Speech and Communication Sciences, Trinity College Dublin, Dublin, Ireland

${ }^{8}$ Discipline of Speech and Language Therapy, School of Health Sciences, National University of Ireland, Galway, Ireland.

Address for correspondence: Dr. Ciara Shiggins, School of Health Sciences, Queen's Building, University of East Anglia, Norwich, Norfolk, UK, NR4 7TJ. E-mail:

c.shiggins@uea.ac.uk

\section{Abstract}

Background: There is growing interest in interventions that promote positive outcomes and well-being for people with aphasia (PWA) and their families, but provision is inconsistent. An asset-based approach, based on the theory of salutogenesis, focuses on what makes you well rather than ill. This approach has been used successfully across a variety of research fields, including health and social care research and practice, and has the potential to provide coherent strategies to support people living successfully with aphasia.

Aim: To explore the relevance and potential of an asset-based approach to promoting and sustaining well-being for PWA and their families, across contexts and cultures.

Methods: Exploratory case studies were carried out in the United Kingdom (UK), Norway, Israel, Ireland and Denmark in a variety of settings. Following an interpretative paradigm, we used qualitative methods including: interviews; appreciative inquiry; group discussions; and participatory action research. Ninety five PWA and 25 family members were asked to identify assets within themselves and their communities that promote, sustain and maintain 
well-being, by responding to: "What makes you feel good / well / healthy?" Data were analysed using thematic analysis.

Findings: An asset-based approach proved to be a powerful means for PWA and family members to explore what helps them live well with aphasia. Key themes were identified: 1) personal journey; 2) helping others; 3) connecting to self; 4) connecting to others; 5) recreation; and 6) personal attributes. Self-identification of assets, within the person and their community, and connections to these, helped PWA and their family members to maintain well-being, overcome barriers and regain confidence. Using this approach, focusing on the person's recognition, activation and mobilisation of assets, could enhance the person's understanding and restore meaning around the stroke and onset of aphasia.

Conclusion: This novel exploratory research demonstrates the relevance and potential across diverse cultural contexts of taking an asset-based approach to promoting and sustaining wellbeing for PWA and their families. Focusing on maintaining connections to these assets and developing meaning around the event, could prevent some of the negative sequela of stroke. The 'patient-professional' relationship must transform into a collaborative partnership, with time and flexibility needed to introduce this approach. Further research should examine how service providers and PWA could develop and operationalise an asset-based approach in clinical and community settings and identify if there is an optimum timing for introducing this approach along the stroke pathway.

Keywords: Aphasia; Stroke; Asset-Based Approach; Well-being; Salutogenesis; Coproduction 


\section{Introduction}

Projected figures for stroke worldwide suggest that there will be 70 million stroke survivors by 2030 (Feigin et al., 2014). Disability attributable to stroke, including aphasia, which affects around a third of stroke survivors (Engelter et al., 2006) will therefore inevitably cause an ever-increasing proportion of the overall worldwide disease burden, with serious implications for health-service planning (Murray et al., 2012). Compared to stroke survivors without aphasia, people with aphasia (PWA) are at greater risk of social isolation (Cruice, Worrall, \& Hickson, 2006; Davidson, Howe, Worrall, Hickson, \& Togher, 2008); changes in social roles and networks (Northcott \& Hilari, 2011; Northcott, Moss, Harrison, \& Hilari, 2016); and emotional disorders, including acute and chronic depression (Baker et al., 2017; Code \& Herrmann, 2003). PWA are at risk of reduced well-being and quality of life because of their communication impairment (Cruice, Worrall, Hickson, \& Murison, 2005; Hilari, 2011; Hilari \& Byng, 2009; LaPointe, 1999; Ross \& Wertz, 2003), with close family members liable to 'third party disability' (Grawburg, Howe, Worrall, \& Scarinci, 2013; Grawburg, Howe, Worrall, \& Scarinci, 2014). Family members of PWA may experience negative changes to their own social participation (Grawburg, Howe, Worrall, \& Scarinci, 2014) possibly due to changes to their own identity (Le Dorze, Salois-Bellerose, Alepins, Croteau, \& Hallé, 2014). For example a family member may adopt a care-giver role (Le Dorze \& Signori, 2010); the PWA may no longer be able to support the family financially; or the family member may develop or experience deterioration of a health condition (Grawburg et al., 2014).

Reduced social connections are a predictive factor of long-term depression (Astrom, Adolfsson, \& Asplund, 1993) and can have further implications for a person's morbidity and mortality. People with post-stroke depression, anxiety and reduced quality of life have increased number of contacts with health services (Gillham, Carpenter, \& Leathley, 2012; 
Murray et al., 2012) and poorer prospects for recovery (Donnellan, Hickey, Hevey, \& O’Neill, 2010). To date the psychological and social needs of people post-stroke and their family members are often not addressed (Royal College of Physicians: Intercollegiate Stroke Working Party, 2016; Stroke Association, 2016; Wren, et al., 2014). Statutory health service resources for PWA are time-constrained, while social care is limited, leading to unmet need in the UK and Ireland (Irish Heart Foundation: Council for Stroke, 2010; Vlachantoni, Shaw, Evandrou, \& Falkingham, 2015). In Israel, inequalities in availability and access to poststroke rehabilitation services is an issue (Zucker et al., 2013). Given the implied increase in prevalence of aphasia and the multidimensional consequences to the individuals and family members, it is imperative that researchers and healthcare professionals continually review the nature and extent of services provided. Increasing the well-being of PWA and family members may have protective effects for health (Steptoe, Deaton, \& Stone, 2015) and should be considered as a key outcome of health and non-health interventions. Here we explore an asset-based approach to promoting and enhancing well-being, which may have relevance and potential for PWA and family members worldwide.

An asset-based approach, based on the theory of salutogenesis (Antonovsky, 1979; Lindstrom, 2005; Mittelmark, Sagy, Eriksson, Bauer, Pelikan, Lindström \& Espnes, 2017), is an orientation to positive change for health and not an intervention in itself. An asset-based approach considers ways of identifying and mobilising individual, group and community resources and capacity (i.e. assets) in order to promote and strengthen factors that enhance and create good health and well-being, rather than focusing on problems and needs (i.e. deficits) (Hopkins \& Rippon, 2015).

In the extensive study of individuals' adaptation to environmental stressors and the origins of health Antonovsky (1979) proposed the Salutogenic Model of Health. He established two key 
components to the theory of salutogenesis: Sense of Coherence (SOC) and General Resistance Resources (GRR), which together can be used to move towards health and wellness. SOC is a flexible global orientation created through life experiences and further developed through: understanding and comprehension (comprehensibility), for example of a significant life event; learning how to manage this (on their own or with others) (manageability), and through these aspects finding a meaning for it (meaningfulness); these aspects can be facilitated through information (Eriksson \& Lindström, 2005). A welldeveloped SOC has a strong correlation to perceived health, mental well-being and quality of life (Antonovsky, 1979; Eriksson \& Lindström, 2007). GRR are resources, either material or non-material that the person possesses, which can be mobilised and used for particular purposes (Antonovsky, 1979; Antonovsky, 1984). These resources may be internal i.e. within the person, for example coping patterns or intelligence, or they may be external i.e. outside of the person, for example connections with people or the community (Eriksson \& Lindström, 2005). People with a higher SOC can self-identify more GRR's (Eriksson \& Lindström, 2005), which in turn can help to create health (Eriksson \& Lindström, 2006).

An asset-based approach has been applied extensively in the areas of community development (Dolezal \& Burns, 2015; Johnson Butterfield, Yeneabat, \& Moxley, 2016; Wu \& Pearce, 2014); education (Chikoko, Naicker, \& Mthiyane, 2015; Missingham, 2017; Ssewamala, Sperber, Zimmerman, \& Karimli, 2010); social welfare (Carter et al., 2016; Culp, 2017; Soaita, Searle, McKee, \& Moore, 2016); and business (Fisher et al., 2009; Tracey, O'Sullivan, Lane, Guy, \& Courtemanche, 2017). For example, the basis of an assetbased approach within the field of community development is to create 'community' by focusing on assets available within the community and connecting these, rather than focusing on shortcomings and deficits (Kretzmann \& McKnight, 1993). The central tenet is that a 
community has assets that can be identified by those in the community themselves, and then developed (Kretzmann \& McKnight, 1993; Mathie \& Cunningham, 2003). Solutions come from within the community itself, with relationships developed or maintained within the community to mobilise assets, while an external body's role is supportive and enabling (Blackman, Buick \& O’Flynn, 2016; Mathie \& Cunningham, 2003; Kretzmann \& McKnight, 1993). Co-production is an important (but contested) element of asset-based approaches (see Bovaird \& Down, 2008). For an illustrative example in the area of community development see Blackman, Buick \& O’Flynn (2016). Positive outcomes from asset-based approaches are evident in relation to poverty alleviation (Ennis \& West, 2013; Toth, 2015; Wu \& Pearce, 2014); access and equality in education (Knight, 2014; McNamara Horvat \& Earl Davis, 2011; Samuelson \& Litzler, 2016); community resilience (Cinderby, Haq, Cambridge, \& Lock, 2016; Paul \& Vogl, 2013); and youth development (Bloomberg, Ganey, Alba, Quintero, \& Alcantara, 2003; Theron \& Malindi, 2010). In the domain of health there has been increasing interest in deploying an asset-based approach over the last decade, particularly in the areas of public and mental health (Boyd, Hayes, Wilson, \& BearsleySmith, 2008; Morgan \& Ziglio, 2007). While a number of studies have addressed well-being in the area of stroke, this is the first study, to our knowledge, that specifically and expli citly explores the application of an asset-based approach in the field of communication disorders.

Taking an asset-based approach in health and rehabilitation faces a number of potential challenges. There may be a risk of misinterpretations or misunderstandings of the conceptual underpinnings of an asset-based approach, for example, that it is an intervention applied by professionals rather than a set of relationships where all parties make resource contributions (e.g. Bovaird \& Downe, 2008). In addition, a failure to connect with conventional health and social care services, or to address competing interests to achieve a power balance for citizens' voices to be heard has been identified as a serious weakness (Friedli, 2013). Indeed Daly and 
Westwood, (2016) argue that as individuals may have varying levels of pre-morbid SOC or assets at their disposal, there is a risk of increasing health inequalities, suggesting that some people may be more suitable for an asset-based approach than others. In order to address some of these challenges Hopkins and Rippon (2015) and Rippon and South (2017) have proposed a 'theory of change' model, including four key elements: 1) Reframing thinking, goals and outcomes; 2) Mapping and describing the range of resources available to individuals and communities; 3) Mobilising assets for a purpose; and 4) Co-producing outcomes (Hopkins \& Rippon, 2015). Rippon and South (2017, p. 14) propose an 'assetbased orientation' as a deliberate mechanism of change threading through these four elements, through which a range of opportunities can be developed, including: articulating the salutogenic concept; developing skills and approaches to support practice and action; incorporating measurement tools; identifying key impacts and benefits.

It is widely accepted that aphasia rehabilitation should focus on more than the impairment, with the overarching goal of empowering PWA to participate in their environments and communities and to maintain and create social connections and new self-identities (SimmonsMackie, 1998; Simmons-Mackie \& Damico, 2007; Chapey et al., 2008). Indeed, the majority of UK Speech and Language Therapists (SLTs) acknowledge that psychological support (93\%) and enhancement of social participation (99\%) are part of their role (Northcott, Simpson, Moss, Ahmed, \& Hilari, 2017). In addition, an international survey of speech and language therapy practice (Hilari et al., 2015) indicated that clinicians considered addressing quality of life outcomes as an important issue. There has been a recent increase in stroke and aphasia research focused on exploring: how a person can live successfully with aphasia (Brown, Davidson, Worrall, \& Howe, 2013; Grohn, Worrall, Simmons-Mackie, Hudson, 2014); self-management (Jones, Riazi, \& Norris, 2013) and a key aspect of self-management, 
self-efficacy (Bandura, 1977). Self-management approaches, for example focus on encouraging people to manage their own health, by being given the knowledge, skills and confidence to do so and to overcome identified barriers to health (Department of Health, 2001). In a recent systematic review (Wray, Clarke, \& Forster, 2018), 46\% of included studies (11 out of 24) showed statistically significant benefits for self-management, although these benefits (on global disability, stroke specific quality of life and activities of daily living) were no longer apparent at 3 or 6 month follow-up. In addition, stroke survivors with aphasia were totally or partially excluded from nearly half of these studies and a further nine studies were unclear if people with aphasia were included. SLTs and other allied health professionals use a variety of approaches to address psychological support in the area of aphasia. These include Solution Focused Therapy / Solution Focused Brief Therapy (Northcott, Burns, Simpson, \& Hilari, 2015); positive psychology (Holland \& Nelson, 2013) and Life Participation Approach to Aphasia (LPAA) (Chapey et al. 2008). These approaches share some conceptual underpinnings with an asset-based approach, for example, building strengths, supporting self-efficacy, and promoting re-engagement and participation by individuals. However, importantly, an asset-based approach, does not solely focus on the individual, but rather aims to harness social capital and promote community connections. Adopting an asset-based orientation implies a fundamental systems shift, including reflection and re-ordering of existing practices towards a participatory, collaborative and opportunitiesbased focus (Rippon \& South, 2017).

There is clearly a need to further develop ways of preventing the negative long-term consequences of stroke (Baker et al., 2017). An asset-based approach may have the potential to shift the conversation to how PWA and family members can create health and well-being in sustainable ways (Lindström \& Eriksson, 2005). This study therefore has undertaken a 
preliminary exploration of the relevance and potential of an asset-based approach to promoting and sustaining well-being for PWA and their families, across contexts and cultures.

\section{Methods}

Settings and participants

Exploratory studies were carried out in five countries, based on an established research collaboration (the Collaboration of Aphasia Trialists). Within each country a convenience sample was used, but with the aim of including PWA with a range of ages, severity of aphasia, and time since onset. Settings varied within and across countries producing a sample, which included a range of contexts, cultures and participants. Location, type of setting and participants are provided in Table 1.

\section{INSERT TABLE ONE ABOUT HERE}

The context for each of the studies set-out in Table 1 reflects the particular culture of the setting, and to some extent the type of activity or expectation of members and the experience of the facilitators for these settings. So, for example, activities pursued in higher education settings reflected the involvement of students, working with PWA for a dissertation at undergraduate or master's level. At Speakeasy (Bury) there is an established culture and wide range of open-ended activity and support from professionals and trained volunteers towards developing strategies, skills and connections for enhancing lives affected by aphasia, with regular conversations around managing 'the way we live'. At the Adler Aphasia Centre (Israel), PWA and family members are involved in all activities including, communication groups, social support groups for family members and individual LPAA intervention; while at Statped (Norway), the conversations happened in the context of week-long group-based 
courses for people who had been living with aphasia for a year or more, drawn from across Norway. The core of these courses is re-building life after the onset of aphasia, with a focus on PWA learning from each other. Study length varied considerably across settings, from one-off discussions to student projects 8 months in duration.

Ethical permissions were sought, as appropriate, for each site. It meant, that in some cases ethical approval was sought through the relevant university ethical committees (University of East Anglia (UEA) (Norwich, UK), National University of Ireland Galway (NUIG) (Ireland) and Trinity College Dublin (TCD) (Ireland)), whereas other sites did not need any further ethical approval because the asset-based conversations were a part of routine activity and formed part of the process of collaboration and consultation (Adler Aphasia Centre, Jerusalem, Israel; Speakeasy, Bury, UK); or because it was not needed due to countryspecific legislation (The University of Southern Denmark (SDU), Odense, Denmark; Statped, Oslo, Norway), however, informed consent was obtained from all participants at these sites.

\section{Design}

We have taken a multiple-case study approach, as appropriate to addressing descriptive and explanatory questions (Yin, 2017) relating to an asset-based approach to well-being after onset of aphasia. We were interested in how participants described their experiences of life after stroke and living with aphasia, or as a family member of someone living with aphasia, from this particular conceptual perspective, gathering experiences and views that provided explanations or counter explanations. We were also interested in asking participants to consider 'how' or 'why' their own or others' experiences may have evolved in particular ways. 
While these 8 settings represent a convenience sample, the diverse nature of the sectors, settings and cultures across the five countries opens up the opportunity for direct and theoretical replication, with sufficient cases to give some confidence in the findings (Yin, 2017).

The theoretical proposition underlying all cases was drawn from our readings and understanding of an asset-based approach, which starts with a focus on 'what makes you feel good, well or healthy?', in line with salutogenic theory (Antonovsky, 1979; Lindström \& Eriksson, 2005). While the basic theoretical perspective was common to all cases, and a general set of guidelines was prepared for the sake of initial common probing questions across all 5 countries, researchers were free to pursue their inquiries with a degree of flexibility, adapting to participants' spontaneous initiatives and communication preferences (e.g. some participants chose non-verbal methods of communication - writing or drawing while others chose to use a combination of verbal and non-verbal etc.)

The research was led at each site by the authors, all with professional qualifications as SLTs (CS, CJ, DO, GP, JI, LH-J, RMcM, SH) or in social work (VS), with extensive experience of working with PWA (7 - 38 years), and each highly experienced in supportive communication techniques. Table 2 sets out approaches, collaborators and methods used in all cases.

\section{Data collection: approaches and methods}

A variety of qualitative approaches underpinned data collection including: Participatory Action Research, which is characterised by repeated cycles of looking, thinking and acting and experiential learning through collaborative research relationships. This requires: 1) 
repeated cycles of action and reflection; 2) dynamic interactions between co-researchers with and without health conditions; and 3) continuous reflection on power relations throughout the research process (Baum, MacDougall, \& Smith, 2006); Appreciative Inquiry (Mathie \& Cunningham, 2005) that aims through discussion and active listening, to discover and build on what is working well in an organisation, group or community, rather than focusing on the problems (Hopkins \& Rippon, 2015); combined with use of semi-structured, one-to-one interviews or group discussions. The underlying concepts pursued in all cases started by exploring the question:

- "What makes you feel good / well / healthy?"

Further questions that were explored in many of the cases were:

- "Which strengths do you find in yourself?"

- "Which are the social connections that make you feel good"

- "What else can help you?"

Activities and materials were flexible and adapted to individuals, groups and settings according to local needs. For example, at Speakeasy the questions: "What makes you feel good / well / healthy?" did not readily resonate with members, so to make the activity more accessible, the analogy 'Planet Aphasia' was used. Speakeasy members who took part were asked "what would you take with you to 'Planet Aphasia' that would help you to feel well; what or who else would be there?” (Appendix 1). Photography was used by one member, who took pictures of "what made him feel well". In Statped, all group participants were shown a 20 minute video $^{1}$ of three people living with aphasia to stimulate conversation and were immediately asked, “what makes you feel well/glad?” In a follow-up discussion with one group, group participants resonated better with the question "what lifts you up?" This 
was conceptualised in an illustration of a person with balloons and group members mapped their assets onto these balloons (Appendix 2). At the UEA (Norwich), NUIG and TCD (Ireland) the iterative nature of reflective group work produced findings and new knowledge over a series of meetings. Supported communication methods were used as appropriate. Data were collected at each site in the native language of the country and translated into English by the relevant author.

\section{INSERT TABLE 2 ABOUT HERE}

Following data collection at each study site authors completed thematic analysis (Braun \& Clarke, 2006, 2013) on their own site's data. Authors were then provided guidelines to record their site's information on participants, data collection methods, key findings and illustrative quotes and narratives using a proforma developed by CS and SH (Appendix 3). Data were sent by all researchers to CS, who collated the information. The process of data organisation, analysis and interpretation is outlined in Figure 1.

\section{INSERT FIGURE 1 ABOUT HERE}

\section{Data analysis}

Data were analysed by three authors $(\mathrm{SH}, \mathrm{RMcM} \& \mathrm{CS})$. A data display was created using each research site's proforma; a visual record was made of each step of data analysis using digital images captured on a smartphone (Appendix 4). Data analysis followed Braun and Clarke's stages for thematic analysis (Braun \& Clarke, 2006, 2013) in an inductive, iterative process. The three co-authors took time to familiarise themselves with the data and to develop an overall initial impression (Braun \& Clarke, 2013). Semantic coding was 
conducted across the data set to identify as many potential codes as possible (Braun \& Clarke, 2013). The co-authors searched for initial themes and patterns across the data set; nine initial themes were identified. Following further discussion data were reviewed to ensure that initial themes reflected and remained true to the overall data set. The initial themes were revised, creating seven candidate themes, and finally, after further consultation between the researchers and further review of the data in relation to the research questions, six final themes were identified. The analytical process was discussed by the three authors and individual workings cross-checked with the other co-authors to improve the rigour of this analytical process.

\section{Findings}

The data collected, with PWA and their family members, across countries and cultures, produced six themes relating to the research question, "what keeps or makes you feel well" including: 1) personal journey; 2) connecting to self; 3) connecting to others; 4) helping others; 5) recreation; and 6) personal attributes. Some of the themes and their sub-themes were interlinked, acting as the mechanisms to sustain and maintain well-being for a person with aphasia (e.g. connections); while others were the consequences of creating a successful life with aphasia (e.g. establishing new identities). Themes and sub-themes are set out below with illustrative narratives and quotations from the data.

\section{1) Personal Journey}

PWA and family members emphasised that each person's experience of aphasia was unique. This was consistent across cultures and contexts. PWA and their family members discussed the 'fragmentation' they experienced after stroke and how re-building life as a stroke survivor takes time and could be hard work. 
Life after stroke was as an ongoing process, during which there were successes, but also challenges to overcome.

“Like falling down a mountain - a steep drop as you've lost people and then gradually you're able to start coming back up" (PWA UEA, Norwich).

"I am on a journey of discovery in life" (PWA Statped, Norway).

PWA said they may face a series of blows: suddenly coming face-to-face with reality ("having to answer the phone"; "trying to wrap presents"); or reaching milestones and anniversaries ("I've survived another year, but I'm terrified") (PWA UEA, Norwich). One person with aphasia described moments in her daily life, where she would be unable to do something that had once been easy for her, for example putting on a bra. These moments would be a stark reminder of a loss of capability, and might lead, on occasions to a temporary "blow up", where feelings were strongly expressed. She called these occasions "stroke moments", and discussed how over time, she came to accept these "stroke moments" and stopped "beating herself up" over them. PWA felt that allowing themselves the time to readjust, while acknowledging the changes to their lives - becoming a person not a patient helped them to maintain well-being.

PWA also discussed how developing an understanding of stroke and aphasia, helped them to re-adjust and feel well. They emphasised the power of information in this process.

"I just laugh and I think how good I've come along with the information" (PWA TCD, Ireland).

PWA felt that information may need to be given multiple times, to allow for it to be processed. Such information needed to be given in accessible forms and required time for discussion and reflection. 


\section{2) Connecting to self}

PWA and their family members described the importance of connecting to a sense of self. Through fragmentation after stroke this sense of self could be lost, but finding ways to rebuild this, helped them to maintain their well-being. For some people, it was connecting to things that gave them a sense of self and identity beyond stroke, for example developing new skills or interests.

"Yeah I just depended on him so the tables turned. I had totally I had to do it all. I had to learn and I learned very fast to do it and I said if anyone thought I couldn't go to the bank or I couldn't do things like that. Women years ago wouldn't be use to writing check or doing any of that stuff. You know a lot of men did it. But anyway I am here in 2017 doing it." (Family member NUIG, Ireland)

For others, it was reconnecting to their 'old self', for example working to re-gain interests or abilities they had before their stroke, such as getting back to work, being able to do housework, getting back to the choir or getting their driving licence back. The key to both of these was feeling 'able' again, feeling that they could participate in activities and achieve. Family members discussed how their own identity had changed, as a result of their family member's stroke. They emphasised a need to have a break from the 'carer role', in order to maintain their own sense of self and an individual identity. They sustained their own wellbeing by doing 'regular' activities.

"When I go out and do 'regular things' that make me feel alive" (Family member, Adler Aphasia Centre, Israel)

Some people even described how they felt that they had become a better person since having their stroke and preferred their new 'self'.

"I think more about others than I used to do. Before I would just talk and talk" (PWA, Statped, Norway)

"I find that I am a softer person than before. And I am more modest" (PWA 2 Statped, Norway) 


\section{3) Connecting to others}

PWA and family members discussed the importance of social connections to maintaining their well-being. Family and friends were often cited as the main people they connected with, in order to feel well.

"Emm so eh I meet up to my friends [...] it is a laugh \{...\} it's a full-time blast" (PWA TCD, Ireland)

I have very good friends and they would ring up and if I wanted anything or maybe bring me for a cup of coffee ..... and I wouldn't be.. I would ask them, as well. Will we go for a walk, will we go for a cup of coffee. Or whatever. So it is everything is a two way not a one way relationship'(Family member, NUIG, Ireland)

However, this was inconsistent across the data. Some people, particularly those who had experienced changes to their social networks or who did not have family, found social connections in their communities. They emphasised the importance of connecting to groups; for example, community groups, charity groups, peer-support and community networks. Some said that they would have no social interaction or opportunities for communication, unless they made an effort to go to these groups. For them, the groups they had connected with were integral to keeping well. For others, it was a chance to meet people beyond their own families, or to meet others with who had experienced stroke and were living with aphasia. People reported that these groups provided an opportunity to build new relationships, and occasionally these relationships were stronger than their previous friendships. These groups also provided structure to the week, providing people with a sense of belonging and a feeling of inclusion.

The family members interviewed also reported that it was important to connect to others, and discussed the importance of being able to talk about their own personal experiences with others who understood. Through organisations for PWA, family members, had developed relationships, experiencing and providing peer-support, based on the experience of living 
with someone who had aphasia.

"Meeting the other wives of PWA, we formed an informal social group, new friends who understand exactly what each of us feels" (Family member, Adler Aphasia Centre, Israel).

PWA and their family members also discussed how important health services, including

SLTs and medical professionals, had been in helping them to maintain their well-being.

"The support we got was from the nurses and the care assistants. They were unbelievable to us. They are our friends" (Family member, NUIG, Ireland)

For some PWA, voluntary organisations, higher education institutions and healthcare services had helped them to develop the skills and confidence to reconnect with others.

"But they organize people to come into that library and tell you about medication that you would need or do ah (.) a physio an acupuncturist would come you know?" (PWA TCD, Ireland).

The importance of animals was also emphasised. Many of the PWA who were interviewed, described how their dog or cat provided them with a sense of connection. One PWA (UEA, Norwich) said that taking up walking dogs for other people since her stroke made her feel good and improved relationships and helped make stronger connections with neighbours.

There was congruence across research sites on the importance of reciprocal relationships. Reciprocity was the mechanism that helped the person to feel well. The se relationships could inspire the PWA, seeing how others were living successfully, as a stroke survivor, and also feeling appreciated and valued by others, for inspiring them.

J: "And ah (.) it was [M] that ah inspired me".

M: "Yes and you inspired me with the month with the children".

M: "So we get great advice from each other yeah" (Two PWA TCD, Ireland).

Such reciprocal relationships had a positive impact on feelings of self-worth and value. However, as PWA pointed out there was continuous work involved in maintaining relationships, not least the need to work harder by finding new ways to communicate. 


\section{4) Helping others}

PWA and their family members reported how helping others, made them feel well. They spoke about the importance of 'giving back' to others. Some discussed how being able to support peers, who were also affected by stroke, had huge benefits for them. People, in particular, emphasised being able to share their experiences and tips with those who were at an earlier stage post-stroke.

"We knew that we would be able to figure out what was wrong where like say a nurse or a care assistant might not have had the time or they mightn't just know what he was trying to do" (Family member NUIG, Ireland)

Many PWA and family members, described how being involved in activities that helped others, made them feel well. They emphasised the importance of making meaningful contributions. For example, two PWA discussed how they had taken specific roles in the organisation of a peer-support group, as the chair and treasurer, and how this had given them a 'sense of purpose'. For these individuals, such roles tapped into expertise and skills from their previous professions. Deploying their expertise, they were able to sustain the peersupport group and go on to achieving charitable status for the group. Some of the group of PWA at UEA had been involved in Conversation Partner Training for a number of years and spoke of how this had been an opportunity to "give something back"; they also felt valued and had become involved in research and other projects. This was also echoed by some participants at NUIG and TCD.

"I find that very good (points to a picture representing the Conversation Partner Programme). You get to meet lovely girls; you see what's happening in the country. See what's happening with younger people. And they would have time to listen" (PWA NUIG, Ireland)

Many PWA described how they liked to be involved in research, either as a participant or in the design, delivery and dissemination. Other PWA provided examples of volunteering or minding grandchildren. 
$T$ (PWA): 'Eh:: every year eh stroke awareness conference is on is on (.)

$S$ (student): Mmhmm

T: Ehh the first week in eh: May.

Rl (Researcher): Mmhmm

$M$ (PWA): $M m h m m$

T: and eh I and my colleagues eh: (.) were sitting inside in eh the room and Stroke awareness (.)

S: Mmhmm

T: and eh (.) eh:: (4) a whole lot a people in front a me and (.)

R1: $M m h m m$

T: and eh I eh I eh aphasia (.)" "He speaks and presents at the Stroke conference each year] (Two PWA TCD, Ireland).

'It's great to have am a project like this [research project] because I think I'm helping other people that are worse than me" (PWA TCD, Ireland). 


\section{5) Recreation}

Recreation and leisure activities helped people to feel well and to live successfully with aphasia. Recreation fell into three sub-themes: places and spaces; active; and passive. PWA and their family members emphasised the importance of places and spaces to their wellbeing. These included being in the outdoors, appreciating and being surrounded by nature, observing the beauty of flowers and feeling sunshine.

People affected by aphasia enjoyed both active and passive recreation. PWA and their family members shared an extensive range of recreational activities that helped them feel well. Examples of active recreation included: engaging in sport and exercise; hiking; eating and drinking; holidays and travel; taking courses; singing in a choir; and aphasia and stroke conferences. Examples of passive recreational activities included: watching sport, TV, art, relaxing and music. The particular activities mentioned were individual to each person's interests, but similar types of recreational activities were discussed across cultures and contexts.

"I cycle down to \{place name\} and talk to people you know, there would be conversation started by about 7.00am, you know, you keep going, somebody there all the time. They say, "Jesus, somebody saw me..told me..yeno keep going. So there's great people there...sport and music are two international languages" (PWA NUIG, Ireland)

Hygge $^{2}$ was a unique recreational activity discussed only by the Danish participants. Finding opportunities to engage in hygge both during activities within and outside their home, e.g. in an aphasia group, or at home with family, friends and pets helped them to feel well. As one participant explains about her aphasia group "and this thing about gathering, that I think is 'hyggeligt' and we used to do that a lot' (PWA SDU, Denmark). Hunting and fishing were recreational activities mentioned only by the Norwegian participants.

For some, this theme was connected to developing, reconnecting or recreating a sense of self. In these activities people did not have to be defined by or associated with stroke or aphasia, 
the focus could be on the activity itself and the personal enjoyment associated with it.

M: "I just go to Tai Chi and eh don't tell them what I have".

R1: "Yeah yeah".

M: “They don't tell me what they have so it's a Tai Chi” (PWA TCD, Ireland)

"We went from bar to bar. I drank soda and didn't talk much. But I was there" (PWA Statped, Norway)

Money and transport could act as barriers to or facilitators of these recreational activities.

\section{6) Personal attributes}

PWA and their family members described how their own personal attributes helped them to feel well. For many, these attributes were inherent to them, prior to the stroke. For example, some PWA described themselves as having a positive attitude and being optimistic.

"I am optimistic, I have joy of life, I am strong, I am a fighter" (PWA Speakeasy, Bury)

However, for others these were attributes that they developed in response to their or a family members' stroke.

"Like if you said to me five years ago this was going to happen. I never thought I was a strong person but I think when you are put into a situation it is either sink or swim. You know. I think we have proved that we are strong women. Like we have a great father and luckily he is so patient and he is worth all the hard work" (Family member NUIG, Ireland)

For another woman with aphasia, it was the encouragement of another PWA that helped her to change her mind-set, to a more positive outlook. He asked her, "If you could do 1000 things before your stroke, do you focus on the 300 things you can't do now, or the 700 things you still can?" This motto became her "lifeline". People felt that a positive attitude helped them to deal with the prognosis of aphasia. Determination and being pro-active were other personal attributes that were discussed by many people affected by aphasia. One PWA described how he gave himself new challenges and goals to achieve, such as doing a skydive or running a marathon (PWA Coventry). He described the link being his determination and 
his ability to remain autonomous and independent. Others discussed how it was the confidence they had developed throughout their life, that helped them to live successfully with aphasia. Some described how the skills they developed with technology and their interest in it, equipped them to using technology to help them live well with aphasia.

"I know to handle situations, I use the I-Pad constantly, I am not shy, I am communicative", (PWA Speakeasy, Bury)

Importantly, there was an emphasis on a need for a sense of humour; to be able to laugh, at times, at their own situation. One group of PWA discussed how it takes a combination of personal attributes, resources and relationships to live successfully with aphasia.

While an asset-based approach does not start with a focus on 'problems' or 'what's wrong', PWA in this study were nevertheless very clear that the ongoing, daily challenges of living with aphasia should be acknowledged as lives were re-assembled. Figure 2 (below) displays an overview of the processes and outcomes produced through analysis and interpretation of findings across cases in our data set.

\section{INSERT FIGURE TWO ABOUT HERE}

This figure suggests a certain forward-moving chronology, which was apparent in many participants' accounts. For example, being involved and contributing might help to address feelings of "isolation" and "uselessness", which might in turn help to develop a sense of survivorship. However, it is also intended to convey a sense of iteration in the 'continuous hard work' needed to scale and overcome the mountains, which would often arise again. Personal attributes - "a cup of courage, hope, self-belief and determination" - are the basis for reconnections, which are supported through relationships to the self and to others, 
restoring meaning to life and enhancing confidence, leading to further connections that make things possible. The figure seeks to illustrate only the experiences shared by the participants included in this study, who, as outlined above constituted a particular convenience sample, while diverse other experiences may have been expressed if other sites or contexts were included.

\section{Discussion}

In this study we explored the relevance and potential of an asset-based approach to promoting and sustaining well-being for PWA and their families in cases across a range of contexts and cultures. The findings of this study would suggest that taking an asset-based approach, underpinned by salutogenic theory, is relevant and has potential for PWA and their family members, with people describing their experiences of life after stroke and living with aphasia from this particular conceptual perspective. The six themes that arose from analysis of participants' experiences and perspectives indicate that personal and interpersonal resources are at the core of positive aspects of their lives. Key themes from the study are discussed in the following section.

Consistent with previous research, taking part in meaningful activities was an important factor to living well with aphasia (Brown et al., 2013; Cruice et al., 2006; Cruice, Worrall, Hickson, \& Murison, 2003). Brown et al. (2013), found that meaningful activities, both communication and non-communication based, helped to "foster pleasure or well-being" (p.146). Also congruent with previous research was the emphasis on and the importance of meaningful relationships (Brown et al., 2013; Cruice et al., 2003; Hinckley, 2006; Holland, 2006). These relationships could involve HCPs as well as family, friends or members of groups (communication and non-communication groups). The importance of relationships 
with healthcare professionals, including SLTs echoes Lawton et al.'s (2018) findings, with participants in this study highlighting the role of HCPs in helping to build confidence and connect or re-connect them to meaningful activities and people. Peer-support groups (Brown et al., 2013) were especially important to our study participants. There is strong evidence that PWA and their family members benefit from peer-support (Brown, Worrall, Davidson, \& Howe, 2011; Van der Gaag, 2005). Participants in this study reported that peer-support groups provided opportunities for reciprocal relationships to develop, and for some people, this linked with the theme of connecting to self - in other words, connecting to others 'in the same boat' was instrumental in helping people to re-connect to some aspect of their own life and to their pre- or post-stroke identity. In the peer-group setting, PWA and family members were able to share information, or support others, and for some, to re-connect with a former role or capability, for example a 'leadership role', by becoming the chairperson of a group. These groups also provided opportunities to give and receive emotional support, further reinforcing the idea of reciprocity. This reciprocity helped the people affected by aphasia (both PWA and their families) to feel valued and gave them a sense of purpose. Helping others, not only being helped is significant for boosting well-being (Oarga, Stavrova, \& Fetchenhauer, 2015). In addition, participants described personal attributes that facilitated them to live successfully with aphasia. The power of positivity (Brown et al, 2012), determination (Holland, 2006) and humour (Simmons-Mackie \& Schultz, 2003) have previously been reported as having a positive impact of living with aphasia and our findings are consistent with the literature. This research reinforces the importance of these personal attributes and how they may be mobilised, both in rehabilitation, but also through personal and community networks. 
Salutogenic theory offers an explanation for the importance of mobilising the person's selfidentified assets. Antonovsky's (1979) Salutogenic Model of Health shows how a person's Sense of Coherence (SOC) and General Resistance Resources (GRRs) can be used to enhance health and well-being. Mobilisation of general resistance resources GRR and development of a SOC can have a positive impact on the person's perceived health and wellbeing (Lindström \& Eriksson, 2005). In our study, PWA and their family members identified a variety of resources, both internal and external, that could be built upon and reinforced. PWA in this study, identified both the resources that helped them to feel well (for example, meeting with others and developing meaningful relationships) and also the mechanisms or processes to achieve this goal (for example, developing confidence or reciprocity). Appreciation of such resources might be immediately apparent, through attending a peersupport group for example, or develop incrementally through immersive experiences, such as being involved in Conversation Partner training or helping develop research in higher education settings. These experiences helped to develop the person's SOC by fostering understanding and comprehension (comprehensibility), helping them to manage (manageability) and develop meaning (meaningfulness) (Antonovsky, 1979), around the stroke and the onset of aphasia.

PWA and their families in this study felt it was essential for people to be provided with appropriate, high quality and accessible information about their condition. The provision of good-quality stroke-related information and post-stroke education is increasingly recognised (Eames, Hoffmann, Worrall, \& Read, 2010; Lowe, Sharma, \& Leathley, 2007). However, a number of barriers to the provision of information have been identified, with the importance of co-ordination, appropriate presentation (Eames et al., 2010), timing and manner of delivering information underlined (Tompkins, Blake, Wambaugh, \& Meigh, 2011). PWA and 
their family members in this study had differing experiences of receiving information. Some had had the opportunity to discuss aphasia with healthcare professionals; however, for others it was only when they met other PWA that they began to understand aphasia and what it meant to live with aphasia. Developing a SOC after emerging into a new and frightening post-stroke world (Ryan, Harrison, Gardiner, \& Jones, 2017), is highly dependent on the person being able to comprehend what has happened, manage themselves and their circumstances, and derive meaning from the event. For many of the participants in this study, the development of SOC occurred in a group setting, where they had the opportunity to discuss their personal experience of stroke openly. Herrmann and Wallesch (1993) and Baker et al. (2017) suggest that people who deny the initial consequences of stroke and the permanency of the disability, are at greater risk for secondary reactive depression. 'Loss of self' and 'loss of person' can interact with depression for PWA (Baker et al, 2017) especially when people are discharged from statutory services and begin to negotiate a new social role with aphasia (Herrmann \& Wallesch, 1993).

\section{Strengths and limitations}

The main strengths of this study are the wide range of countries, cultures and contexts of the participating PWA and family members. Previous research in the area of 'living successfully with aphasia' was conducted mainly in the United States of America and Australia and with smaller sample sizes. Conversations between researchers and PWA and family members in this study were in-depth and iterative, and involved an on-going process in some research sites. Unlike studies by Holland (2006) and Hinckley (2006), for example our study sample was not based on 'living successfully with aphasia' criteria, but included a broad range of participants who were simply encouraged to share their experiences of and perspectives on living with aphasia. 
In this exploratory study we adopted a flexible approach to the methods of data collection, with our inquiry framed in a number of textual and / or visual ways. While this suggests potential inconsistencies in the overall approach, it was instrumental, along with skilful use of supported communication techniques in enabling us to adapt to the communicative needs of PWA whose impairments ranged from mild to severe.

However, orientating PWA or family members towards an asset-based approach, rather than a deficit focus, was often challenging. While some participants easily connected with the concepts entailed in an asset-based approach, others took more time to 'reframe' (Hopkins \& Rippon, 2015; Rippon \& South, 2017). Connecting to asset-informed concepts was easier where there were long-established relationships between PWA and researcher / author or where special support services to family members are available (e.g. Bury, Speakeasy, Adler Aphasia Centre), or where PWA had already been involved in research development, teaching and co-production of knowledge (e.g. UEA, Norwich; TCD, Ireland; NUIG, Ireland). However, even then it took some participant-researcher collaborations time to establish a frame of reference, with PWA, family and researcher / students equally affected (e.g. UEA, Norwich). The experience was inconsistent across research sites for family member participants, with some connecting easily to the concepts.

Across the health literature, the relationship between the healthcare professional and the patient, has been shown to have an impact on outcomes (Krupnick et al., 1996; Martin, Garske, \& Davis, 2000), including in the area of aphasia (Lawton et al., 2018). In this study, (re)framing this relationship into a collaborative partnership was important to exploring and developing an understanding of experiences and perspectives relative to an asset-based approach. Our study was undertaken in a broad range of settings, many non-clinical, however 
Galvin and colleagues (2018), in their work on humanising healthcare, provide some suggestions on how this could be accomplished, within busy healthcare settings. In our experience of conducting this research we suggest that taking the time to complete the stages as outlined by Hopkins \& Rippon (2015) and Rippon \& South (2017, p.14) are essential to equalising this relationship and orientating the person towards assets, rather than deficits. Our experience supports Rippon and South's (2017) proposal that an 'asset-based orientation' needs to be a deliberate mechanism of change, where the salutogenic concept should be clearly articulated and skills to support action in this approach purposively developed. Morgan et al.'s (2014) critique that challenges can arise in an asset-based approach through 'misinterpretations and misunderstandings' is supported in our study, which highlighted the need for skilled facilitators to overcome barriers to understanding, and to help develop collaborative work. Flexibility in how this approach was introduced to PWA and their family members was essential. Although exploring different means of introducing these concepts poses a methodological limitation to this study, using creative approaches helped some PWA and their family members to connect with the concepts and made them culturally appropriate, which mitigated the chance of 'misinterpretation and misunderstandings' to arise. Also, as this was an exploratory study the authors learned from the different sites across the course of the study about implementing a novel approach in the field of aphasia. Examples include using the analogy of 'Planet Aphasia' (Speakeasy, Bury), or capitalising on descriptions and sharing of asset-rich experiences among participating PWA in group discussion. While recording of and reflection on experiences (e.g. UEA, Norwich; NUIG and TCD, Ireland; Speakeasy, Bury) produced rich and evolving data, this was a lengthy process, potentially outside the bounds of possibility in a time-starved health care setting. Using the most appropriate method, for the individual PWA and their family members, all participants identified an asset (something or someone), that kept them feeling well. In the context of 
group discussions, occasionally a peer's discussion of their assets, stimulated another person to identify their own and therefore, highlighting the potential influence of group dynamics on the process. The authors acknowledge that while all the participants in this research were able to self-identify assets, for others interacting co-morbid difficulties or low-mood may make it difficult to identify assets.

\section{Conclusion}

Several limitations and their implications for future research should be noted. All the PWA in this study had chronic aphasia (> 6 months post-stroke). In order to better understand the feasibility of implementing an asset-based approach with PWA and their family members along the entire stroke pathway, it would be important to include participants in the acute stage, in-patient rehabilitation, Early Supported Discharge, and pre-discharge. The majority of participants included in the current study were either, accessing healthcare services, connected to a group, educational institutions or involved in their community. Therefore, these participants are not necessarily representative of the full range of people who are living with aphasia, for example those who do not wish to meet others who live with aphasia or participate in groups, or those who are more withdrawn or isolated. In addition, we did not include the full range of stakeholders that are involved in the rehabilitation process in our study, for example no SLTs or commissioners were interviewed. In further work with clinicians, we would need to explore how this approach could translate into clinical practice and how current evidence-based interventions, as well as novel future innovations, could be used within this approach. The full range of opinions, across the stroke pathway, would be invaluable to better understand how this approach could be implemented and operationalised within current healthcare systems. 
Despite SLTs / SLPs considering psychological support and quality of life outcomes as part of their role (Hilari et al., 2015; Northcott et al., 2017), the psychological needs of a person post-stroke and their caregivers has to date been broadly neglected (Stroke Association, 2016). An asset-based approach has the potential to offer a new orientation to positive change, providing strategic and systematic theoretical underpinnings to complement approaches currently used to increase participation and address the negative sequelae of stroke and aphasia.

Adopting an asset-based approach requires radical re-framing and adjustment of expectations and action by health and social care professionals, voluntary services and patients alike. For example, the focus on individual and community 'assets' rather than problems, deficits and needs requires fundamental re-working of individual and organisational practices (for a wealth of up-to-date examples see https://www.nurturedevelopment.org/). However, we feel that despite the upfront investment needed in 're-framing' and re-working existing practices this innovative approach may well fulfil the requirements of affordability and sustainability of a 'frugal innovation' (see Weyrauch \& Herstatt, 2017), producing a robust and durable framework for delivering well-being outcomes for PWA and families.

In our study we found that the application of an asset-based approach was relevant and had potential for PWA and their family members, across countries and cultures. Exploring the lived experience of aphasia, using this approach, has proven feasible. Our research supports Brown et al. (2012), in calling for a transformation of rehabilitation for PWA, where the central focus is on how people can remain connected to the things that keep them well, rather than a focus on needs or deficits. While the concept of living successfully with aphasia is not a new one (Brown et al., 2012; Holland, 2006), an asset-based approach provides and strong 
theoretical basis and a practical framework (e.g. Hopkins and Ripon, 2015; Ripon and South, 2017) to enable this transformation.

How an asset-based approach for people with aphasia and families could be implemented and operationalised, within health, social care and third sector organisations, necessitates further research. A failure to work within the current systems of health and social care is often cited as a weakness of this approach (Friedli, 2013). Future research should explore how an assetbased approach could be implemented along the stroke pathway, and include all key stakeholders.

Acknowledgements: We acknowledge funding from COST Cooperation in Science and Technology, Collaboration of Aphasia Trialists (ISCH COST Action IS1208) and Vibeke \& Victor Bloch's Foundation.

In addition, we acknowledge the invaluable contributions of people with aphasia and their family members: the Aphasia Research Collaboration (UEA); Speakeasy (Bury, UK); Aphasia United (conference delegates); CONNECTing group (TCD); Statped (Norway); and all participants in this research, as well as student and staff collaborators: Dr. Irene Walsh, Caoimhe Loftus and Emma Jones (Trinity College Dublin), Maja Højbjerg Horn and Anne Kirstine Frost Frederiksen (University of Southern Denmark), Felicity Stephenson, Nicola Milbourn, Olivia Pembery, Rachel Pillips, Tasnim Morrison, Zoe Mardell (University of East Anglia), Elizabeth O’ Brien and Clodagh Mc Greevy (National University of Ireland Galway), Mali Gil (Loewenstein Rehabilitation Hospital, Israel)

\section{Conflicts of interest: None}

\section{References}

Afasiforbundet i Norge [The National Aphasia Association in Norway]. (2012). Afasihverdag. [Aphasia in everyday life.] [Video clip]. Retrieved from: https://afasi.no/2012/09/09/filmen-afasihverdag/ (Accessed on: 28/09/18)

Antonovsky, A. (1979). Health, Stress \& Coping. Michigan: Jossey-Bass.

Antonovsky, A. (1984). The sense of coherence as a determinant of health. In J. Matarazzo (Ed.), Behavioral Health A Handbook of Health Enhancement and Disease Prevention (pp. 114-129). New York: John Wiley.

Astrom, M., Adolfsson, R., \& Asplund, K. (1993). in Stroke Patients A 3-Year Longitudinal Study. Stroke, 24(7), 976-982. http://doi.org/10.1161/01.STR.24.7.976

Baker, C., Worrall, L., Rose, M., Hudson, K., Ryan, B., \& O’Byrne, L. (2017). A systematic review of rehabilitation interventions to prevent and treat depression in post-stroke 
aphasia. Disability and Rehabilitation, $0(0), 1-23$.

http://doi.org/10.1080/09638288.2017.1315181

Bandura, A. (1977). Self-efficacy: Toward a unifying theory of behavioral change. American Psychological Association, 84(2), 191-215. http://doi.org/10.1037/0033-295X.84.2.191

Baum, F., MacDougall, C., \& Smith, D. (2006). Participatory action research. Journal of Epidemiology and Community Health, 60(10), 854-857. http://doi.org/10.1136/jech.2004.028662

Blackman, D. A., Buick, F., \& O'Flynn, J. (2016). From engaging to enabling: Could an asset-based approach transform Indigenous affairs?. Environment and Planning $C$ : Government and Policy, 34(8), 1632-1651.

Bloomberg, L., Ganey, A., Alba, V., Quintero, G., \& Alcantara, L. A. (2003). Chicano-Latino Youth Leadership Institute: An asset-based program for youth. American Journal of Health Behavior, 27(SUPPL. 1).

Bovaird, T. and Downe, J. (2008) Innovation in Public Engagement and CoProduction of Services. Policy Paper to Department of Communities and Local

Government. Cardiff: Cardiff Business School.

Boyd, C. P., Hayes, L., Wilson, R. L., \& Bearsley-Smith, C. (2008). Harnessing the social capital of rural communities for youth mental health: An asset-based community development framework. Australian Journal of Rural Health, 16(4), 189-193. http://doi.org/10.1111/j.1440-1584.2008.00996.x

Braun, V., \& Clarke, V. (2006). Using thematic analysis in psychology. Qualitative Research in Psychology, 3(May 2015), 77-101. http://doi.org/10.1191/1478088706qp063oa

Braun, V., \& Clarke, V. (2013). Successful Qualitative Research: a practical guide for beginners. London: SAGE Publications, Inc.

Brown, K., Davidson, B., Worrall, L. E., \& Howe, T. (2013). "Making a good time": The role of friendship in living successfully with aphasia. International Journal of SpeechLanguage Pathology, 15(2). http://doi.org/10.3109/17549507.2012.692814

Brown, K., Worrall, L. E., Davidson, B., \& Howe, T. (2011). Living successfully with aphasia: A qualitative meta-analysis of the perspectives of individuals with aphasia, family members, and speech-language pathologists. International Journal of SpeechLanguage Pathology, 14(2), 141-155.

Carter, E. W., Blustein, C. L., Bumble, J. L., Harvey, S., Henderson, L. M., \& McMillan, E. D. (2016). Engaging communities in identifying local strategies for expanding integrated employment during and after high school. American Journal on Intellectual and Developmental Disabilities, 121(5), 398-418. http://doi.org/10.1352/1944-7558121.5.398

Chapey, R. (2008) (editor). Language intervention strategies in aphasia and related neurogenic communication disorders. Philadelphia: Wolters Kluwer Health/Lippincott Williams \& Wilkins.

Chikoko, V., Naicker, I., \& Mthiyane, S. (2015). School leadership practices that work in areas of multiple deprivation in South Africa. Educational Management Administration \& Leadership, 43(3), 452-467. http://doi.org/10.1177/1741143215570186

Cinderby, S., Haq, G., Cambridge, H., \& Lock, K. (2016). Building community resilience: can everyone enjoy a good life? Local Environment, 21(10), 1252-1270. http://doi.org/10.1080/13549839.2015.1100597

Code, C., \& Herrmann, M. (2003). The relevance of emotional and psychosocial factors in aphasia to rehabilitation. Neuropsychological Rehabilitation, 13(1-2), 109-132. http://doi.org/10.1080/09602010244000291

Collins English Dictionary. (2018). In [online]. Available at: https://www.collinsdictionary.com/dictionary/english/hygge (Accessed: 28/09/18) 
Cruice, M., Worrall, L., \& Hickson, L. (2006). Quantifying aphasic people's social lives in the context of non-aphasic peers. Aphasiology, 20(February 2015), 1210-1225. http://doi.org/10.1080/02687030600790136

Cruice, M., Worrall, L., Hickson, L., \& Murison, R. (2003). Finding a focus for quality of life with aphasia: Social and emotional health, and psychological well-being. Aphasiology, 17(4), 333-353. http://doi.org/10.1080/02687030244000707

Cruice, M., Worrall, L., Hickson, L., \& Murison, R. (2005). Measuring quality of life: Comparing family members' and friends' ratings with those of their aphasic partners. Aphasiology, 19(2), 111-129. http://doi.org/10.1080/02687030444000651

Culp, B. (2017). ?Illegitimate? Bodies in Legitimate Times: Life, Liberty, and the Pursuit of Movement. Quest, 69(2), 143-156. http://doi.org/10.1080/00336297.2017.1287578

Daly, M., \& Westwood, S. U. E. (2016). Asset-based approaches, older people and social care : an analysis and critique. Forum Keele University, 1-18.

Davidson, B., Howe, T., Worrall, L., Hickson, L., \& Togher, L. (2008). Social Participation for Older People with Aphasia: The Impact of Communication Disability on Friendships. Topics in Stroke Rehabilitation, 15(4), 325-340. http://doi.org/10.1310/tsr1504-325

Department of Health. (2001). The expert patient: a new approach to chronic disease management for the twenty-first century. London: Department of Health.

http://webarchive.nationalarchives.gov.uk/20120511062115/http://www.dh.gov.uk/prod_con sum_dh/groups/dh_digitalassets/@dh/@en/documents/digitalasset/dh_4018578.pdf (Accessed on: 2/07/18).

Dolezal, C., \& Burns, P. M. (2015). ABCD to CBT: asset-based community development's potential for community-based tourism. Development in Practice, 25(1), 133-142. http://doi.org/10.1080/09614524.2015.982075

Donnellan, C., Hickey, A., Hevey, D., \& O’Neill, D. (2010). Effect of mood symptoms on recovery one year after stroke. International Journal of Geriatric Psychiatry, 25(12), 1288-1295. http://doi.org/10.1002/gps.2482

Eames, S., Hoffmann, T., Worrall, L., \& Read, S. (2010). Stroke Patients' and Carers' Perception of Barriers to Accessing Stroke Information. Topics in Stroke Rehabilitation, 17(2), 69-78. http://doi.org/10.1310/tsr1702-69

Engelter, S. T., Gostynski, M., Papa, S., Frei, M., Born, C., Ajdacic-Gross, V., ... Lyrer, P. a. (2006). Epidemiology of aphasia attributable to first ischemic stroke: Incidence, severity, fluency, etiology, and thrombolysis. Stroke, 37(6), 1379-1384. http://doi.org/10.1161/01.STR.0000221815.64093.8c

Ennis, G., \& West, D. (2013). Using social network analysis in community development practice and research: A case study. Community Development Journal, 48(1), 40-57. http://doi.org/10.1093/cdj/bss013

Eriksson, M., \& Lindström, B. (2005). Validity of Antonovsky's sense of coherence scale: a systematic review. Journal of Epidemiology \& Community Health, 59(6), 460-466.

Eriksson, M., \& Lindström, B. (2006). Antonovsky's sense of coherence scale and the relation with health: a systematic review. Journal of Epidemiology \& Community Health, 60(5), 376-381.

Eriksson, M., \& Lindström, B. (2007). Antonovsky's sense of coherence scale and its relation with quality of life: a systematic review. J Epidemiol Community Health, 61, 938-944. http://doi.org/10.1136/jech.2006.056028

Feigin, V. L., Forouzanfar, M. H., Krishnamurthi, R., Mensah, G. A., Connor, M., Bennett, D. A., ... Naghavi, M. (2014). Global and regional burden of stroke during 1990-2010: Findings from the Global Burden of Disease Study 2010. The Lancet, 383(9913), 245255. http://doi.org/10.1016/S0140-6736(13)61953-4 
Fisher, K., Geenen, J., Jurcevic, M., McClintock, K., Davis, G., Greenen, J., ... Davis, G. (2009). Applying assett-based community development as a strategy for CSR: a Canadian perspective on a win-win for stakeholders and SMEs. Business Ethics A European Review, 18(1), 66-82. http://doi.org/10.1111/j.1467-8608.2009.01549.x

Friedli, L. (2013). "What we"ve tried, hasn't worked' : the politics of assets based public health ${ }^{1}$. Critical Public Health, 23(2), 131-145. http://doi.org/10.1080/09581596.2012.748882

Galvin, K. T., Sloan, C., Cowdell, F., Ellis-Hill, C., Pound, C., Watson, R., ... Brooks, S. (2018). Facilitating a dedicated focus on the human dimensions of care in practice settings: Development of a new humanised care assessment tool (HCAT) to sensitise care. Nursing Inquiry, (February), e12235. http://doi.org/10.1111/nin.12235

Gillham, S., Carpenter, M., \& Leathley, M. (2012). Psychological care after stroke: economic modelling of a clinical psychology led team approach. London.

Grawburg, M., Howe, T., Worrall, L., \& Scarinci, N. (2013). Third-party disability in family members of people with aphasia: A systematic review. Disability and Rehabilitation, 35(16). http://doi.org/10.3109/09638288.2012.735341

Grawburg, M., Howe, T., Worrall, L., \& Scarinci, N. (2014). Describing the impact of aphasia on close family members using the ICF framework. Disability and Rehabilitation, 36(14), 1184-1195. http://doi.org/10.3109/09638288.2013.834984

Grawburg, M., Howe, T., Worrall, L., \& Scarinci, N. (2014). Describing the impact of aphasia on close family members using the ICF framework. Disability and Rehabilitation, 36(14), 1184-1195. http://doi.org/10.3109/09638288.2013.834984\r10.1080/02687038.2013.768330; Grawburg, M., Howe, T., Worrall, L., Scarinci, N., Third-party disability in family members of people with aphasia - A systematic review (2013) Disabil Rehabil, 35, pp. 1324-1341; Bakas, T., Kroenke, K., Plue, L.D., Outcomes among family caregivers of aphasic versus nonaphasic stroke survivors (2006) Rehabil Nurs, 31, pp. 33-42; Le Dorze, G., Signori, F.H., Needs, barriers and facilitators experienced by spouses of people with aphasia (

Grohn, B., Worrall, L., Simmons-Mackie, N., Hudson, K. (2014). Living successfully with aphasia during the first year post-stroke: A longitudinal qualitative study. Aphasiology, 28(12), 1405-1425.

Herrmann, M., \& Wallesch, C. W. (1993). Depressive changes in stroke patients. Disability and Rehabilitation, 15(2), 55-66. http://doi.org/10.3109/09638289309165873

Hilari, K. (2011). The impact of stroke: are people with aphasia different to those without? Disability and Rehabilitation, 33(3), 211-218. http://doi.org/10.3109/09638288.2010.508829

Hilari, K., \& Byng, S. (2009). Health-related quality of life in people with severe aphasia. International Journal of Language \& Communication Disorders / Royal College of Speech \& Language Therapists, 44(2), 193-205. http://doi.org/10.1080/13682820802008820

Hilari, K., Klippi, A., Constantinidou, F., Horton, S., Penn, C., Raymer, A., ... Worrall, L. (2015). An International Perspective on Quality of Life in Aphasia: A Survey of Clinician Views and Practices from Sixteen Countries. Folia Phoniatrica et Logopaedica, 67(3), 119-130. http://doi.org/10.1159/000434748

Hinckley, J. J. (2006). Successfully with Stroke and Aphasia, 13(1), 25-36.

Holland, A. (2006). Living Successfully with Aphasia: Three variations on the Theme. Topics in Stroke Rehabilitation, 13(1), 44-51.

Holland, A. (2013) Positive Psychology. In. Holland, A, Nelson, R. (2013) Counseling in Communication Disorders: A Wellness Perspective ( $2^{\text {nd }}$ Ed.) 
Holland, A. L., \& Nelson, R. L. (2013). Counseling in communication disorders: A wellness perspective. Plymouth: Plural Publishing.

Hopkins, T., \& Rippon, S. (2015). Head, Hands and Heart: Asset-Based Approaches in Health Care.

Irish Heart Foundation: Council for Stroke. (2010). National Clinical Guidelines and Recommendations for the Care of People with Stroke and Transient Ischaemic Attack.

Johnson Butterfield, A. K., Yeneabat, M., \& Moxley, D. P. (2016). "Now I Know My ABCDs": Asset-based community development with school children in Ethiopia. Children and Schools, 38(4), 199-207. http://doi.org/10.1093/cs/cdw031

Jones, F., Riazi, A., \& Norris, M. (2013). Self-management after stroke: Time for some more questions? Disability and Rehabilitation, 35(5), 257-264. http://doi.org/10.3109/09638288.2012.691938

Knight. (2014). Toward a Relational Perspective The Contextual Patterns of Disclosure as Coping. Harvard Educational Review, 84(4), 433-468.

Kretzmann, J. P., \& McKnight, J. L. (1993) Building Communities from the Inside Out: A Path Toward Finding and Mobilizing a Community's Assets. Chicago, IL: ACTA Publications.

Krupnick, J. L., Sotsky, S. M., Simmens, S., Moyer, J., Elkin, I., Watkins, J., \& Pilkonis, P. A. (1996). The role of the therapeutic alliance in psychotherapy and pharmacotherapy outcome: Findings in the National Institute of Mental Health Treatment of Depression Collaborative Research Program. Journal of Consulting and Clinical Psychology, 64(3), 532-539. http://doi.org/10.1037/0022-006X.64.3.532

LaPointe, L. (1999). Quality of Life with Aphasia. Seminars in Speech and Langauge, 20(1), $5-17$.

Lawton, M., Sage, K., Haddock, G., Conroy, P., \& Serrant, L. (2018). Speech and language therapists' perspectives of therapeutic alliance construction and maintenance in aphasia rehabilitation post-stroke. International Journal of Language \& Communication Disorders, 550-563. http://doi.org/10.1111/1460-6984.12368

Le Dorze, G., Salois-Bellerose, É., Alepins, M., Croteau, C., \& Hallé, M. C. (2014). A description of the personal and environmental determinants of participation several years post-stroke according to the views of people who have aphasia. Aphasiology, 28(4), 421-439. http://doi.org/10.1080/02687038.2013.869305

Le Dorze, G., \& Signori, F. H. (2010). Needs, barriers and facilitators experienced by spouses of people with aphasia. Disability and Rehabilitation, 32(13), 1073-1087. http://doi.org/10.3109/09638280903374121

Lindstrom, B. (2005). Salutogenesis. Journal of Epidemiology \& Community Health, 59(6), 440-442. http://doi.org/10.1136/jech.2005.034777

Lindström, B., \& Eriksson, M. (2005). Salutogenesis. Journal of Epidemiology and Community Health, 59(6), 440-442. http://doi.org/10.1136/jech.2005.034777

Lowe, D. B., Sharma, A. K., \& Leathley, M. J. (2007). The CareFile project: A feasibility study to examine the effects of an individualised information booklet on patients after stroke. Age and Ageing, 36(1), 83-89. http://doi.org/10.1093/ageing/afl 145

MacLeod, M. A., \& Emejulu, A. (2014). Neoliberalism With a Community Face? A Critical Analysis of Asset-Based Community Development in Scotland. Journal of Community Practice, 22(4), 430-450. http://doi.org/10.1080/10705422.2014.959147

Martin, D. J., Garske, J. P., \& Davis, M. K. (2000). Relation of the Therapeutic Alliance With Outcome and Other Variables : A Meta-Analytic Review, 68(3), 438-450.

Mathie, A., \& Cunningham, G. (2003). From clients to citizens: Asset-based community development as a strategy for community-driven development. Development in practice, 13(5), 474-486. 
Mathie, A., \& Cunningham, G. (2005). Who is Driving Development? Reflections on the Transformative Potential of Asset-based Community Development. Canadian Journal of Development Studies, 26(1), 175-186.

http://doi.org/10.1080/02255189.2005.9669031

McNamara Horvat, E., \& Earl Davis, J. (2011). Schools as Sites for Transformation:

Exploring the Contribution of Habitus. Youth \& Society, 43(1), 142-170.

http://doi.org/10.1177/0044118X09358846

Missingham, B. D. (2017). Asset-based learning and the pedagogy of community development. Community Development, 5330(June), 1-12. http://doi.org/10.1080/15575330.2017.1291528

Mittelmark, M., Sagy, S., Eriksson, M., Bauer, G., Pelikan, J., Lindström, B., \& Espnes, G. (2017). The handbook of salutogenesis. Switzerland: Springer.

Morgan, A., \& Ziglio, E. (2007). Revitalising the evidence base for public health: an assets model. Promotion \& Education, 14(2_suppl), 17-22. http://doi.org/10.1177/10253823070140020701x

Morgan, A. (2014). Revisiting the asset model: a clarification of ideas and terms. IUHPE Global Health Promotion, 21, (2), 3-6.

Murray, C. J. L., Vos, T., Lozano, R., Naghavi, M., Flaxman, A. D., Michaud, C., ... Lopez, A. D. (2012). Disability-adjusted life years (DALYs) for 291 diseases and injuries in 21 regions, 1990-2010: A systematic analysis for the Global Burden of Disease Study 2010. The Lancet, 380(9859), 2197-2223. http://doi.org/10.1016/S0140-6736(12)61689-4

Northcott, S., Burns, K., Simpson, A., \& Hilari, K. (2015). "Living with Aphasia the Best Way I Can": A Feasibility Study Exploring Solution-Focused Brief Therapy for People with Aphasia. Folia Phoniatrica et Logopaedica, 67(3), 156-167. http://doi.org/10.1159/000439217

Northcott, S., \& Hilari, K. (2011). Why do people lose their friends after a stroke? International Journal of Language \& Communication Disorders, 46(5), 524-534. http://doi.org/10.1111/j.1460-6984.2011.00079.x

Northcott, S., Moss, B., Harrison, K., \& Hilari, K. (2016). A systematic review of the impact of stroke on social support and social networks: associated factors and patterns of change. Clinical Rehabilitation, 30(8). http://doi.org/10.1177/0269215515602136

Northcott, S., Simpson, A., Moss, B., Ahmed, N., \& Hilari, K. (2017). How do speech-andlanguage therapists address the psychosocial well-being of people with aphasia? Results of a UK online survey. International Journal of Language and Communication Disorders, 52(3), 356-373. http://doi.org/10.1111/1460-6984.12278

Oarga, C., Stavrova, O., \& Fetchenhauer, D. (2015). When and why is helping others good for well-being? The role of belief in reciprocity and conformity to society's expectations. European Journal of Social Psychology, 45(2), 242-254. http://doi.org/10.1002/ejsp.2092

Paul, B. G., \& Vogl, C. R. (2013). Organic shrimp aquaculture for sustainable household livelihoods in Bangladesh. Ocean and Coastal Management, 71, 1-12. http://doi.org/10.1016/j.ocecoaman.2012.10.007

Rippon, S., \& South, J. (2017). Promoting Asset Based Approaches for Health and Wellbeing Exploring a Theory of Change and Challenges in Evaluation Promoting Asset BAsed APProAcHes for HeAltH And WellBeing contents 3 Promoting Asset BAsed APProAcHes for HeAltH And WellBeing, (November). Retrieved from http://eprints.leedsbeckett.ac.uk/4497/7/Promoting asset based approaches Nov 2017.pdf

Ross, K., \& Wertz, R. (2003). Quality of life with and without aphasia. Aphasiology, 17(February), 355-364. http://doi.org/10.1080/02687030244000716

Royal College of Physicians: Intercollegiate Stroke Working Party. (2016). National clinical 
guideline for stroke. Retrieved from

https://www.strokeaudit.org/SupportFiles/Documents/Guidelines/2016-National-

Clinical-Guideline-for-Stroke-5t-(1).aspx

Ryan, T., Harrison, M., Gardiner, C., \& Jones, A. (2017). Challenges in building interpersonal care in organized hospital stroke units: The perspectives of stroke survivors, family caregivers and the multidisciplinary team. Journal of Advanced Nursing, 73(10), 2351-2360. http://doi.org/10.1111/jan.13313

Samuelson, C. C., \& Litzler, E. (2016). Community Cultural Wealth: An Assets-Based Approach to Persistence of Engineering Students of Color. Journal of Engineering Education, 105(1), 93-117. http://doi.org/10.1002/jee.20110

Simmons-mackie, N. (1998). A solution to the discharge dilemma in aphasia: Social approaches to aphasia management. Aphasiology, 12(3), 231-239. http://doi.org/10.1080/02687039808249451

Simmons-Mackie, N., \& Schultz, M. (2003). The role of humour in therapy for aphasia. Aphasiology, 17(8), 751-766.

Soaita, A. M., Searle, B. A., McKee, K., \& Moore, T. (2016). Becoming a landlord: strategies of property-based welfare in the private rental sector in Great Britain. Housing Studies, 3037(June), 1-25. http://doi.org/10.1080/02673037.2016.1228855

Ssewamala, F. M., Sperber, E., Zimmerman, J. M., \& Karimli, L. (2010). The potential of asset-based development strategies for poverty alleviation in Sub-Saharan Africa. International Journal of Social Welfare, 19(4), 433-443. http://doi.org/10.1111/j.14682397.2010.00738.x

Steptoe, A., Deaton, A., \& Stone, A. A. (2015). Subjective wellbeing, health, and ageing. The Lancet, 385(9968), 640-648. http://doi.org/10.1016/S0140-6736(13)61489-0

Stroke Association. (2016). State of the Nation Stroke statistics. http://doi.org/10.2307/j.ctt80ggr

Theron, L. C., \& Malindi, M. J. (2010). Resilient street youth: a qualitative South African study. Journal of Youth Studies, 13(6), 717-736. http://doi.org/10.1080/13676261003801796

Tompkins, C. a., Blake, M. T., Wambaugh, J., \& Meigh, K. (2011). A novel, implicit treatment for language comprehension processes in right hemisphere brain damage: Phase I data. Aphasiology, 25(6-7), 789-799. http://doi.org/10.1080/02687038.2010.539784

Toth, R. (2015). Traps and thresholds in pastoralist mobility. American Journal of Agricultural Economics, 97(1), 315-332. http://doi.org/10.1093/ajae/aau064

Tracey, S., O?Sullivan, T. L., Lane, D. E., Guy, E., \& Courtemanche, J. (2017). Promoting Resilience Using an Asset-Based Approach to Business Continuity Planning. SAGE Open, 7(2), 215824401770671. http://doi.org/10.1177/2158244017706712

van der Gaag A., S. L. (2005). Therapy and support services for people with long-term stroke. Clinical Rehabilitation, 372-380.

Vlachantoni, A., Shaw, R. J., Evandrou, M., \& Falkingham, J. (2015). The determinants of receiving social care in later life in England The determinants of receiving social care in later life in England, (February). http://doi.org/10.1017/S0144686X1300072X

Weyrauch, T., \& Herstatt, C. (2017). What is frugal innovation? Three defining criteria. Journal of Frugal Innovation, 2(1), 1. http://doi.org/10.1186/s40669-016-0005-y

Wray, F., Clarke, D., \& Forster, A. (2018). Post-stroke self-management interventions: a systematic review of effectiveness and investigation of the inclusion of stroke survivors with aphasia. Disability and Rehabilitation, 40(11), 1237-1251. http://doi.org/10.1080/09638288.2017.1294206

Wren, M, A., Gillespie, P., Smith, S., Kearns, K., Crichton, S., Parkin, D., \& Hickey, A. 
(2014). Towards Earlier Discharge, Better Outcomes, Lower Cost: Stroke Rehabilitation in Ireland. Dublin. Retrieved from 9780707003719

Wu, M.-Y., \& Pearce, P. L. (2014). Asset-based community development as applied to tourism in Tibet. Tourism Geographies, 16(3), 438-456.

http://doi.org/10.1080/14616688.2013.824502

Yin, R.K. (2017). Case study research and applications: Design and methods. London: Sage publications $\left(6^{\text {th }} \mathrm{Ed}\right.$.)

Zucker, I., Laxer, I., Rasooli, I., Han, S., Cohen, A., \& Shohat, T. (2013). Regional gaps in the provision of inpatient rehabilitation services for the elderly in Israel: Results of a national survey. Israel Journal of Health Policy Research, 2(1), 1-7.

http://doi.org/10.1186/2045-4015-2-27 